\title{
Development of intelligent model for personalized guidance on wheelchair tilt and recline usage for people with spinal cord injury: Methodology and preliminary report
}

\author{
Jicheng Fu, PhD; ${ }^{*}$ Maria Jones, PT, PhD; ${ }^{2}$ Yih-Kuen Jan, PT, PhD ${ }^{\mathbf{3}}$ \\ ${ }^{1}$ Department of Computer Science, University of Central Oklahoma, Edmond, OK; ${ }^{2}$ Department of Rehabilitation Sci- \\ ences, University of Oklahoma Health Sciences Center, Oklahoma City, OK, ${ }^{3}$ Department of Kinesiology and Commu- \\ nity Health, University of Illinois at Urbana-Champaign, Champaign, IL
}

\begin{abstract}
Wheelchair tilt and recline functions are two of the most desirable features for relieving seating pressure to decrease the risk of pressure ulcers. The effective guidance on wheelchair tilt and recline usage is therefore critical to pressure ulcer prevention. The aim of this study was to demonstrate the feasibility of using machine learning techniques to construct an intelligent model to provide personalized guidance to individuals with spinal cord injury (SCI). The motivation stems from the clinical evidence that the requirements of individuals vary greatly and that no universal guidance on tilt and recline usage could possibly satisfy all individuals with SCI. We explored all aspects involved in constructing the intelligent model and proposed approaches tailored to suit the characteristics of this preliminary study, such as modeling research participants, using machine learning techniques to construct the intelligent model, and evaluating the performance of the intelligent model. We further improved the intelligent model's prediction accuracy by developing a two-phase feature selection algorithm to identify important attributes. Experimental results demonstrated that our approaches showed promise: they could effectively construct the intelligent model, evaluate its performance, and refine the participant model so that the intelligent model's prediction accuracy was significantly improved.
\end{abstract}

Key words: artificial neural network, C4.5 decision tree, machine learning, pressure ulcer, random forest, skin blood flow, skin perfusion, spinal cord injury, support vector machine, wheelchair tilt and recline.

\section{INTRODUCTION}

Pressure ulcers are a significant threat to the quality of life for people with spinal cord injury (SCI). The current research evidence shows that the majority of individuals with SCI (up to 85\%) will develop at least one pressure ulcer during their lifetimes [1-2]. Pressure ulcers can lead to pain and infection and account for 7 to 8 percent of deaths in the SCI population [1]. In addition, pressure ulcers often result in prolonged hospital stays, rehospitalization, and ever increasing treatment costs. The United States alone spends about $\$ 11$ billion annually on the treatment of pressure ulcers [3]. Among the expenditure, 12 percent is attributed to the treatment of pressure ulcers for people with SCI.

\footnotetext{
Abbreviations: 10 Fold $=10$-fold crossvalidation, $\mathrm{ANN}=$ artificial neural network, $\mathrm{CFS}=$ correlation-based feature subset selection, LDF = laser Doppler flowmetry, LOO = leave-oneparticipant-out, $\mathrm{RF}=$ random forest, $\mathrm{RSE}=$ resubstitution estimator, $\mathrm{SCI}=$ spinal cord injury, $\mathrm{SVM}=$ support vector machine.

*Address all correspondence to Jicheng Fu, PhD; Department of Computer Science, University of Central Oklahoma, 100 North University Dr, Edmond, OK 73034; 405974-5704. Email: ifu@uco.edu
}

http://dx.doi.org/10.1682/JRRD.2013.09.0199 
A key to pressure ulcer prevention is pressure relief [4]. Clinical evidence shows that turning patients regularly (e.g., every 2-4 h) can lower the incidence of pressure ulcers [3], and sitting-induced pressure can be relieved by performing wheelchair tilt and recline functions [3,5-6]. The wheelchair tilt function refers to the adjustment of the seat orientation angle (measured against the ground) while keeping the seat-to-back angle unchanged. The wheelchair recline function refers to changing the seat-to-back angle while maintaining the seat orientation angle (measured against the ground). In its position paper [4], the Rehabilitation Engineering and Assistive Technology Society of North America summarized existing study results and concluded that the best pressure reduction effects are achieved if the wheelchair tilt and recline functions are used in combination.

In practice, clinicians typically recommend the same tilt and recline setting to all patients. Such kinds of uniform recommendations may not be effective because the clinical evidence clearly illustrates that the requirements of individuals with SCI vary greatly [5,7]. Hence, personalized guidance for people with SCI can be more desirable and beneficial. Although statistical methods are commonly used to model biomedical problems, they are found less capable of finding patterns, dealing with data that may contain noise, or analyzing nonlinear and dependent data [8-9]. Machine learning techniques, on the other hand, have played an increasingly important role in bioinformatics for classifying and mining data. Such techniques can capture patterns based on examples (i.e., training data) even though the underlying nature, principles, and/or probability distributions may not be clear [10]. Successful applications have been found in genomics and proteomics [11-12], cancer detection [1315], heart rate variability (HRV) analysis [16], etc. For example, Chou et al. used artificial neural networks to diagnose breast cancer and achieved 98 percent accuracy [15].

In our case, we used machine learning techniques to investigate skin blood flow response to wheelchair tilt and recline usage. The rationale is that skin blood flow response to loading pressure has been widely used to determine the effectiveness of seating conditions $[6,17-$ 19]. The purpose of periodically performing wheelchair tilt and recline is to develop reactive hyperemia to reperfuse the ischemic tissues because prolonged tissue ischemia has been determined to be critical factor leading to pressure ulcers. The reactive hyperemia is a vital response to ischemia [20]. Insufficient reactive hyper- emic responses may cause pressure ulcers to form [20 22]. Hence, our goal is to build an intelligent model to predict whether a given tilt and recline setting would be favorable for skin blood flow increase for an individual, i.e., personalized guidance.

We explored all aspects involved in constructing the intelligent model and proposed approaches tailored to suit the characteristics of this study. The participants with SCI were modeled with their demographic information, neurological functions, and SCI injury history. Such a model suits this preliminary study in that it contains important attributes associated with SCI individuals, while its information can be obtained easily. Then, the participant model together with the wheelchair tilt and recline settings served as the input to the intelligent model. Based on the inputs, the intelligent model classified tilt and recline settings into two classes: the positive class (that is favorable for increasing a participant's skin blood flow) and the negative class (otherwise). To prepare training data for constructing the intelligent model, we designed a threshold-based method to reduce the possibility of falsely classifying negative/neutral cases as positive cases (i.e., false positive).

Based on training data, we investigated four wellknown machine learning techniques (i.e., classification algorithms) to construct the intelligent model, namely, artificial neural network (ANN) [10], support vector machine (SVM) [23], C4.5 decision tree [24], and random forest (RF) [25]. ANN is renowned for its learning capability, adaptability, and ability to generalize [26]. SVM is widely considered one of the best classification algorithms that tends to return high-quality results by finding the global optimum $[23,27]$. C4.5 performs classification by constructing a decision tree. Each node in the decision tree selects an attribute (e.g., age $>50$ ) to divide the training data. As a result, the classification results are more informative because the decision trees can explain how the classification works. In contrast, RF employs a set of decision trees in its learning process. The output is the majority vote among all the decision trees.

After an intelligent model is built, a critical issue is how to evaluate its performance. The popular approaches, such as resubstitution estimator (RSE) [11] and 10-fold crossvalidation (10Fold) [28], lack the ability to evaluate how well the intelligent model performs if a new participant is given because each research participant is associated with six data instances in this study. We applied a leave-one-participant-out (LOO) approach to estimate 
the expected error rate for the intelligent model. This approach overcomes the weakness of RSE and 10Fold by always testing the intelligent model with a new (unseen) participant.

To further improve the intelligent model's prediction ability, we developed a two-phase feature selection algorithm for identifying important attributes as input variables for the intelligent model. The first phase uses a well-known feature selection algorithm, correlationbased feature subset selection (CFS) [29], to determine an initial subset of attributes. Then, the second phase iteratively enumerates the remaining attributes and adds to the subset the ones that can improve the prediction accuracy the most. The benefit of this two-phase algorithm is that it constantly generates a model that performs better than the one generated by using CFS alone.

Experimental results showed that our proposed approaches could effectively construct the intelligent model, evaluate the intelligent model's performance, and identify a subset of important attributes to significantly improve its predication accuracy. This study is an extension of our previous study [17], which provides informative guidance on wheelchair tilt and recline usage using statistical methods on the mean skin perfusion data for clinically recommended tilt and recline settings. This study complements our previous study by providing personalized guidance to individuals with SCI and paves the way to a more comprehensive model in the subsequent study. To the best of our knowledge, no such intelligent models are currently available. The experience learned from this study will lay the groundwork for future investigators in this new research direction.

\section{METHODS}

\section{Participants and Procedures}

We performed a study to investigate skin blood flow response to seating conditions by means of wheelchair tilt and recline. Eleven adult wheelchair users with SCI participated in the study. Consent forms were collected prior to the experiments. The protocol consisted of six clinically recommended testing conditions, the combination of three tilt angles at $15^{\circ}, 25^{\circ}$, and $35^{\circ}$, and two recline angles at $100^{\circ}$ and $120^{\circ}$. Each testing condition was divided into three equal periods starting with a $5 \mathrm{~min}$ sitting-induced ischemic period without performing tilt or recline, followed by a $5 \mathrm{~min}$ pressure reduction period by means of tilt and recline, and concluding with a $5 \mathrm{~min}$ washout period, which was designed to allow participants to fully recover skin blood flow and reduce the carryover effect [6]. The details of protocols are described in our previous study [17]. Laser Doppler flowmetry (LDF) (Periflux System 5001, Perimed; Järfälla, Sweden) was used to measure skin blood flow $\left(\mathrm{mL}_{\mathrm{LDF}} / \mathrm{min} / 100 \mathrm{~g}\right.$ tissue) over the skin on the right ischial tuberosity. Ischial tuberosity was chosen because it is the most common site for sitting-induced pressure ulcers [5]. The data for skin blood flow are reported in our previous study [17].

\section{Problem Modeling}

Personalized guidance on wheelchair usage relies on the ability to determine whether a given tilt and recline setting will result in skin blood flow increase for individual wheelchair users with SCI. This ability is empowered by an intelligent model that was constructed to establish the relationship between wheelchair tilt and recline settings and the resulting skin perfusion response for individuals. Specifically, it takes as input an individual's personal information and a tilt and recline setting and determines whether the given tilt and recline setting will be favorable for increasing the individual's skin blood flow.

Formally, the functionality of the intelligent model can be represented by a function $f$ (Equation 1):

$$
f(p, t, r) \rightarrow\{0,1\}
$$

where $p$ is a person with SCI and $t$ and $r$ are tilt and recline angles, respectively. Given a person $p$, the goal of function $f$ is to determine whether the tilt and recline setting $\langle t, r\rangle$ will result in skin perfusion increase (denoted by 1 ; otherwise, 0 ). Therefore, to determine $f$, we need to address three issues, namely, (1) how to define skin perfusion increase/decrease, (2) how to model a research participant, and (3) how to prepare the training data, based on which we can use machine learning techniques to induce the function $f$.

\section{Threshold-Based Definition of Skin Perfusion Changes}

To address the first issue listed in the "Problem Modeling" section, i.e., how to define skin perfusion increase/ decrease, we use Equation 2 as follows:

$$
\beta=b_{1} / b_{0}
$$


where $b_{0}$ was the skin perfusion measured during the sitting-induced ischemic period (i.e., the first $5 \mathrm{~min}$ in a testing condition) and $b_{1}$ was the skin perfusion measured during the following pressure reduction period (i.e., the next $5 \mathrm{~min}$ in the testing condition) caused by performing wheelchair tilt and recline functions. Therefore, if $\beta>1.00$, it means that the skin perfusion is increased as a result of performing wheelchair tilt and recline. The use of 1.00 as the threshold, however, may result in false positives. For example, if $\beta$ is only slightly over 1.00 (e.g., $1.01)$, then it is uncertain whether this is a true positive result because noise or measurement precision may play a role in such marginally positive cases. In this study, besides 1.00 , we also tested two other thresholds, namely, 1.10 and 1.15 . When the threshold becomes bigger, the positive cases should have relatively larger $b_{1}$ to make $\beta$ greater than the threshold (see Equation 2). Hence, bigger thresholds can help reduce the possibility of false positives.

\section{Modeling Research Participants}

To address the second issue listed in the "Problem Modeling" section, we modeled a research participant with attributes listed in Table 1. The information was obtained from the information forms, which were filled out by research participants prior to enrolling in this study. We attempted to model research participants with these "handy" attributes so that the intelligent model would be easy to use. Among these attributes, the body mass index $(b)$ and age at onset of SCI $(\tilde{a})$ are derived attributes. Body mass index $(b)$ is calculated by $b=w / h^{2}$ and age at onset of SCI ( $\tilde{a})$ is calculated by $\tilde{a}=a-d$. Attributes of smoking $(s)$, alcohol $(o)$, exercise (e), and

Table 1.

Attributes of research participant.

\begin{tabular}{lr}
\hline Category & \multicolumn{1}{c}{ Attributes } \\
\hline Basic Attributes & Age $(a)$, gender $(g)$, Height $(h)$, \\
Weight $(w)$, Body mass index $(b)$ \\
$\begin{array}{l}\text { Neurological Attributes } \\
\text { Spinal Injury History }\end{array}$ & $\begin{array}{l}\text { Level of injury }(l) \text {, Completeness }(c) \\
\text { Duration of injury }(d) \text {, Age at onset } \\
\text { of SCI }(\tilde{a})\end{array}$ \\
$\begin{array}{l}\text { Social/Behavioral Attri- } \\
\text { butes }\end{array}$ & Smoking $(s)$, Alcohol $(o)$, \\
Skin/Wound History & Exercise $(e)$, \\
\hline \hline
\end{tabular}

pressure ulcer history $(u)$ have binary values, namely "yes" or "no." Combining all these attributes, we obtain a model for a participant as Equation 3:

$$
\langle a, g, h, w, b, l, c, d, \tilde{a}, s, o, e, u\rangle \in P
$$

where $P$ is the set of participants.

\section{Preparing Training Data}

In this section, we address the third issue listed in the "Problem Modeling" section, i.e., how to prepare training data. As shown in Equation 1, the function $f(p, t, r) \rightarrow$ $\{0,1\}$ can map an input $\langle p, t, r\rangle$ into one of the two classes, i.e., either 1 or 0 representing whether the skin perfusion increases or not. Correspondingly, we organized the training data into a set of input-output pairs with each pair being in the form of $(\langle p, t, r\rangle, o p t)$, where opt is the output that is either 1 or 0 . Then, we used this set of input-output pairs to train machine learning algorithms to discover the relationship between the inputs and outputs. In Equation 3, a person $p$ is modeled with $\langle a, g, h, w, b, l$, $c, d, \tilde{a}, s, o, e, u\rangle$. Therefore, an input instance $\langle p, t, r\rangle$ is modeled as $\langle a, g, h, w, b, l, c, d, \tilde{a}, s, o, e, u, t, r\rangle$. The output is either 1 or 0 . Since $\beta_{i}$ in Equation 2 is available in each testing condition $\left\langle t_{i}, r_{i}\right\rangle$ for a participant $p$, we labeled an input instance $\left\langle a_{i}, g_{i}, h_{i}, w_{i}, b_{i}, l_{i}, c_{i}, d_{i}, \tilde{a}_{i}, s_{i}\right.$, $\left.o_{i}, e_{i}, u_{i}, t_{i}, r_{i}\right\rangle$ as 1 if and only if the corresponding $\beta_{i}>\tau$, where $\tau$ is the threshold, whose value can be $1.00,1.10$, or 1.15; otherwise, we labeled it as 0 . Formally, the training data were organized in a set $D_{T}$ of input-output pairs (Equation 4):

$$
\begin{gathered}
D_{T}=\left\{\left(x_{i}, y_{i}\right) \mid x_{i}=\left\langle a_{i}, g_{i}, h_{i}, w_{i}, b_{i}, l_{i}, c_{i}, d_{i}, \tilde{a}_{i}, s_{i}, o_{i}, e_{i}, u_{i},\right.\right. \\
\left.\left.t_{i}, r_{i}\right\rangle, y_{i} \in\{0,1\}, \text { and } 1 \leq i \leq N\right\},
\end{gathered}
$$

where $x_{i}$ is the input, $y_{i}$ is the output (i.e., class), and $N$ is the total number of instances of training data.

\section{Constructing the Intelligent Model}

Based on the problem model, we tackled three critical issues that were involved in constructing the intelligent model. First, we evaluated four popular machine learning algorithms to construct the model, namely, ANN [10], SVM [23], C4.5 decision tree [24], and RF [25]. Second, we used an LOO approach to estimate the expected error rate when using the intelligent model to predict new (unseen) data. This approach overcomes the weakness of the popular approaches, such as RSE [11] 
and 10Fold [28], which lack the ability to organize testing data meaningfully into unseen research participants. Third, we developed a two-phase feature selection algorithm to determine a subset of important attributes that enables a classifier to significantly improve its prediction accuracy.

\section{Machine Learning Algorithms Used in This Study}

As shown in Equation 1, the function $f(p, t, r) \rightarrow\{0$, 1 ) classifies an input instance as either 1 or 0 . Hence, we use classification algorithms (i.e., classifiers) to determine the function $f$ because a classifier can be trained to determine the membership of an input instance, i.e., to which class the instance belongs. With Weka [30], we could use four widely used classifiers to determine the function $f$ and determine which algorithm provided the best classification accuracy and generalization ability.

ANN is known for its excellent capability to learn functions from examples (training data) [10]. An ANN has a layered network structure, in which the processing units (i.e., neurons) are arranged in layers. Neurons in adjacent layers can communicate with each other by sending and receiving signals through the weighted connections. The input/output behavior of a neuron is defined by its internal activation function, which accumulates the input signals and then calculates the outputs. Once the network structure is determined, the learning process proceeds in iterations by tuning the weights of the connections using a training algorithm, such as the best-known back-propagation algorithm [31] that applies the gradient descendent rule to tune weights as shown in Equation 5:

$$
w_{i j}=w_{i j}-\eta \frac{\partial E}{\partial w_{i j}}
$$

where $w_{i j}$ is the weight associated with the connection between neurons $i$ and $j ; \eta>0$ is a constant learning rate; and $E=\sum_{v \in D_{T}}\left(l_{v}-o_{v}\right)^{2}$ is the output error with $v$ being the input data, $l_{v}$ being the expected output (i.e., label), and $o_{v}$ being the actual output from ANN.

SVM is a powerful machine learning algorithm, which models each piece of sample data as a point in a space. It then employs hyperplanes to separate positive data from the negative ones. The hyperplane can be represented with Equation 6:

$$
w \cdot x-b=0,
$$

where $w$ is a normal vector, $x$ is a data point, "." is the dot product between two vectors, and $b /|w|$ is the offset of the hyperplane. In case data are linearly inseparable, SVM maps data into a higher dimensional space by using a kernel function, i.e., replacing the dot products with a kernel function. As a result, the data may become separable in the higher dimensional space. In our study, we used the polynomial kernel function as shown in Equation 7, which is known for improving the classification accuracy.

$$
K(x, y)=(x \cdot y+1)^{p},
$$

where $p$ is the parameter to be set by the users. SVM usually returns the global optimal result, which is a big advantage over ANN since ANN is prone to being stuck at local optima.

C4.5 is another well-known classification algorithm that generates a decision tree for classification. It uses Entropy $(S)=\sum_{i=1}^{n} p_{i} \log 1 / p_{i}$ to measure whether an attribute can effectively split data. Here, $S$ is a partition of sample data into subsets: $s_{1}, s_{2} \ldots s_{n}$. $p_{i}$ represents the probability that a data instance belongs to $s_{i}$. Intuitively, the decision is difficult to make if an attribute splits data equally, i.e., 50 percent vs 50 percent, which has the highest entropy, 1 . Hence, C4.5 splits data by selecting the attribute that has the smallest entropy so that the information gain is biggest, i.e., decisions can be easily made. The advantage of C4.5 over ANN and SVM is that the decision tree explains how the classification works and, therefore, provides a better understanding of the underlying process that generates the results.

$\mathrm{RF}$ is a newer classification algorithm, which consists of a number of decision trees (i.e., forest). When a decision tree attempts to select an attribute to split data, it chooses from a random subset of the attributes. The randomness is controlled by RF such that all trees share the same distribution in the forest [25]. The output is a majority vote among the outputs of individual decision trees. RF is widely used because it is efficient and accurate. Unlike C4.5, the output of RF does not provide information explaining how the classification works.

\section{Estimating Expected Prediction Accuracy}

We first used two popular approaches, RSE [11] and 10Fold [28], to estimate the prediction error rate for the intelligent model. RSE uses all the training data to train the classification algorithms and then uses the same set of data to test how well the classification algorithms classify 
these data. Although RSE is easy to use, it may suffer from serious overfitting problems [11]. Overfitting occurs when a classification algorithm can only classify existing data well but fails to predict new data correctly.

10 Fold is widely used to mitigate the overfitting effects [28]. In 10Fold, data are equally partitioned into 10 mutually exclusive sets. One set is chosen as the testing set, and the remaining 9 sets are used as training data. This process repeats 10 times with each time being for one fold. Then, the averaged classification accuracy over the 10 folds is used as the final classification accuracy.

However, because of the randomness of partitioning data into 10 folds, 10Fold cannot estimate how well the intelligent model works if an unseen participant is given. Based on the characteristics of our training data, we applied an LOO approach. Specifically, we left out data (in 6 tilt and recline testing conditions) associated with a participant as testing data and used data of the remaining 10 participants as training data. The major advantage of LOO over 10Fold is that the testing data belong to the same participant and, therefore, the intelligent model is always tested by a new (unseen) participant. Hence, this approach is more meaningful and more accurate in evaluating the generalization ability of the intelligent model.

\section{Two-Phase Feature Selection Algorithm for Identifying Important Attributes}

We proposed a two-phase feature selection algorithm in an attempt to remove irrelevant or redundant attributes. The rationale is that irrelevant or redundant attributes may negatively affect the learning quality [32]. It took two phases to determine a subset of attributes from the training data set $D_{T}$ (see Equation 4). In the first phase, we used a well-known feature selection algorithm, CFS [29], to select a subset of the attributes that are important predictor variables to the intelligent model. This subset is called the initial core attributes set.

Then, the second phase tried to add more attributes to the core attribute set to further improve the intelligent model's prediction accuracy, as shown in Figure 1. The inputs include a classifier $(\mathrm{clf})$, the current core attribute set $\left(S_{\text {core }}\right)$, the remaining attribute set $\left(S_{\text {candidates }}\right)$, and the current maximum prediction accuracy (max) achieved by clf on $S_{\text {core }}$. The algorithm iteratively adds the remaining attributes to the core attributes set, one attribute at a time (lines 2 and 3). The function Evaluate(clf, $S_{\text {core }} \cup\{v\}$ ) returns the prediction accuracy rate by performing the LOO experiment with classifier $c l f$ on the subset of attri-

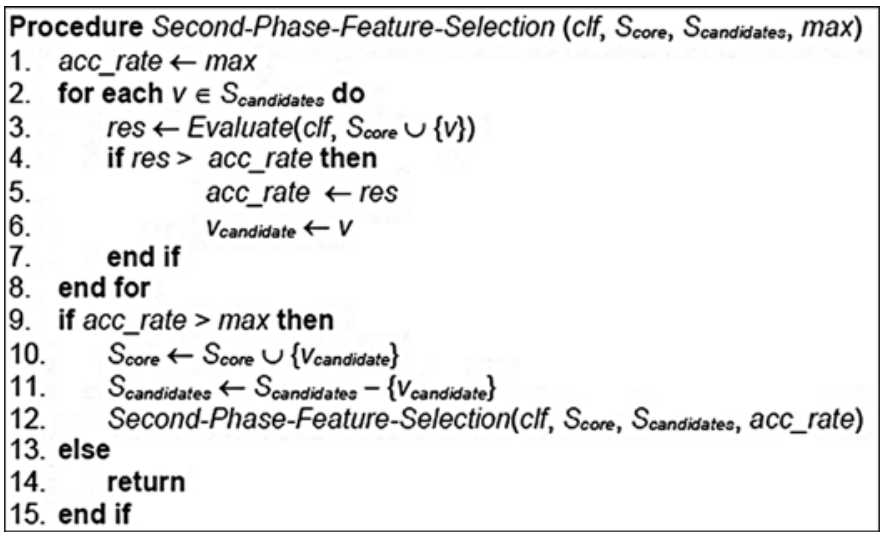

Figure 1.

Second phase algorithm.

butes $S_{\text {core }} \cup\{v\}$ (line 3 ). If the accuracy rate is increased (line 4$)$, the candidate attribute $v_{\text {candidate }}$ and accuracy rate acc_rate are updated (lines 5 and 6). After all the remaining attributes are tested, the attribute that results in the most significant improvement on prediction accuracy is added to the core attributes set (lines 9-11). Then, the algorithm repeats the same process by recursively calling Second-Phase-Feature-Selection algorithm (line 12). The algorithm terminates when no attributes can be added to improve the prediction accuracy (line 14). We chose this recursive approach because an attribute appearing irrelevant in the current iteration may become relevant in the next iteration by combining with other attributes [32].

\section{RESULTS}

\section{Resubstitution Estimator}

Table 2(a) shows the results on the experiment with the resubstitution estimator. All the classification algorithms performed well on all three categories (corresponding to the three thresholds). The lowest accuracy was still above 87 percent made by $\mathrm{C} 4.5$ with the threshold of 1.10. SVM correctly classified the whole training set in all three categories. ANN and RF also achieved accuracies above 93 percent. The network structure for ANN was 15-8-2, i.e., the network consisted of 3 layers with 15 neurons in the input layer, 8 neurons in the hidden layer, and 2 neurons in the output layer. 
Table 2.

Experimental results with all attributes (percent accuracy).

(a) Resubstitution Estimator

\begin{tabular}{lrrr}
\hline \multirow{2}{*}{ Algorithm } & \multicolumn{3}{c}{ Threshold } \\
\cline { 2 - 4 } & $\mathbf{1 . 0 0}$ & $\mathbf{1 . 1 0}$ & $\mathbf{1 . 1 5}$ \\
\hline ANN & 100.00 & 95.31 & 93.75 \\
SVM & 100.00 & 100.00 & 100.00 \\
C4.5 & 95.31 & 87.50 & 93.75 \\
RF & 98.44 & 100.00 & 100.00
\end{tabular}

(b) 10-Fold Crossvalidation

\begin{tabular}{lccc}
\hline \multirow{2}{*}{ Algorithm } & \multicolumn{3}{c}{ Threshold } \\
\cline { 2 - 4 } & $\mathbf{1 . 0 0}$ & $\mathbf{1 . 1 0}$ & $\mathbf{1 . 1 5}$ \\
\hline ANN & 78.13 & 76.56 & 68.75 \\
SVM & 78.13 & 64.06 & 73.44 \\
C4.5 & 82.81 & 70.31 & 73.44 \\
RF & 87.50 & 79.69 & 79.69 \\
\hline
\end{tabular}

(c) Leave-One-Participant-Out

\begin{tabular}{lrcr}
\hline \multirow{2}{*}{ Algorithm } & \multicolumn{3}{c}{ Threshold } \\
\cline { 2 - 4 } & $\mathbf{1 . 0 0}$ & $\mathbf{1 . 1 0}$ & $\mathbf{1 . 1 5}$ \\
\hline ANN & 53.79 & 41.66 & 42.42 \\
SVM & 56.82 & 39.39 & 47.73 \\
C4.5 & 35.61 & 41.66 & 57.58 \\
RF & 37.12 & 52.27 & 50.76
\end{tabular}

$\overline{\mathrm{ANN}}=$ artificial neural network, $\mathrm{RF}=$ random forest, $\mathrm{SVM}=$ support vector machine.

\section{0-Fold Crossvalidation}

Table 2(b) shows the results for the experiment of 10Fold. The accuracy rates of all the classifiers dropped in all three categories compared with the results in Table 2(a). The decrease in classification accuracy suggests that overfitting did occur. Among all the classifiers, RF achieved the best performance in all three categories. Its classification accuracies dropped below 90 percent. Meanwhile, C4.5 performed relatively better than SVM and ANN.

\section{Leave-One-Participant-Out}

Table 2(c) shows the results for the experiment of LOO. The accuracy rates of all classifiers dropped sharply compared with the results in Table 2(a) and (b). With the threshold of 1.00, SVM performed the best with an accuracy of 56.82 percent. In the category of $1.10, \mathrm{RF}$ achieved the highest classification accuracy: 52.27 percent. In the category of $1.15, \mathrm{C} 4.5$ achieved the best performance with an accuracy of 57.58 percent. Figure 2 presents an intuitive illustration of how overfitting affected the classification algorithms. In the sequence of RSE, 10Fold, and LOO, the classification accuracies dropped for all classification algorithms in all three categories.

In fact, if we look into the outputs of C4.5, we find that not all attributes were used in its decision trees. Figure 3 shows one of the decision trees generated in the experiment of LOO. Besides tilt and recline, it only took four other attributes, namely, smoking, exercise, alcohol, and height. This observation suggested that only a subset of the attributes may be useful in constructing the intelligent model.

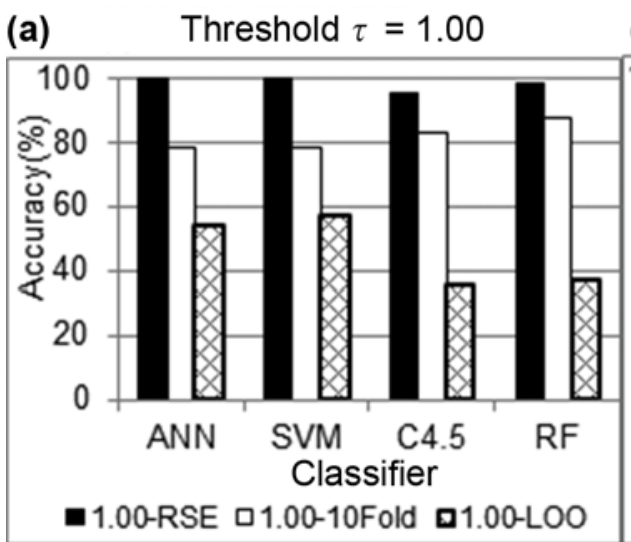

(b) Threshold $\tau=1.10$

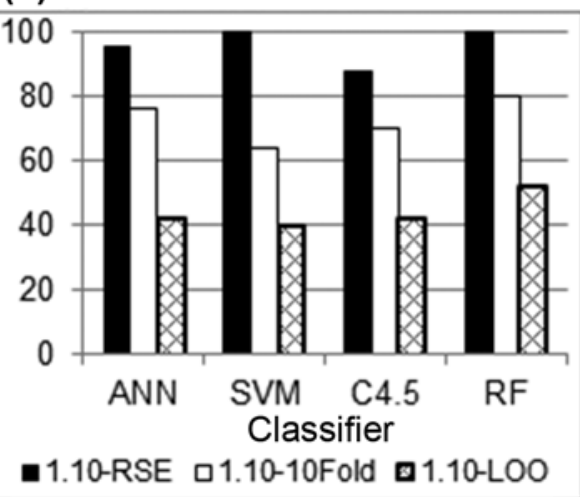

(c) Threshold $\tau=1.15$

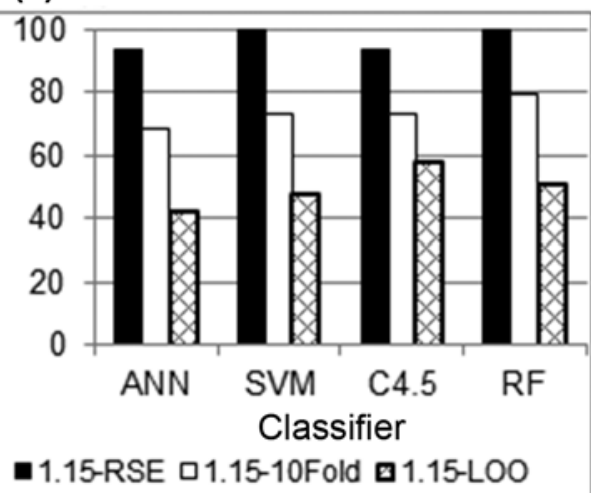

Figure 2.

Accuracy comparisons among resubstitution estimator (RSE), 10-fold cross-validation (10Fold), and leave-one-participant-out (LOO) for thresholds (a) 1.00, (b) 1.10, and (c) 1.15. ANN = artificial neural network, RF = random forest, SVM = support vector machine. 


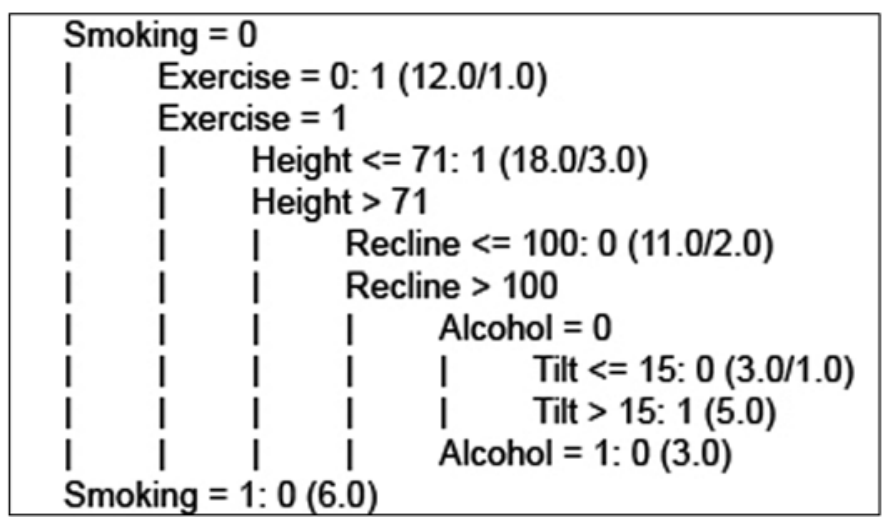

Figure 3.

Sample output of C4.5 decision free.

\section{Two-Phase Feature Selection Algorithm for Identifying Important Attributes}

After running the CFS algorithm in the first phase, we obtained two attributes, level of injury and smoking, as the core attributes. Hence, the core attributes set was initialized to be \{level of injury, smoking\}.

In the second phase, our algorithm automatically ran each classifier by following the steps specified in Figure 1.
The results are shown in Table 3. Comparing Table 3 with Table 2(c), we can see that all the classifiers significantly improved their classification accuracy. Especially, SVM achieved the highest classification accuracy, i.e., 78.79 percent when the threshold 1.15 was applied. Figure 4 presents an intuitive illustration regarding how much the classification accuracy was improved when a subset of the attributes (i.e., core attributes) was used as opposed to the entire attributes. The improvement was significant for all the algorithms in all three categories.

\section{DISCUSSION}

This study demonstrated the feasibility of using machine learning techniques to construct an intelligent model that can provide personalized guidance on wheelchair tilt and recline usage for individual wheelchair users with SCI. Based on the characteristics of our study, we proposed approaches to model human participants, process training data, construct the intelligent model, estimate expected prediction error rates, and refine the participant model by identifying a subset of important

Table 3.

Classification accuracy based on subsets of attributes.

\begin{tabular}{|c|c|c|c|c|c|c|}
\hline \multirow{3}{*}{ Algorithm } & \multicolumn{6}{|c|}{ Threshold } \\
\hline & \multicolumn{2}{|c|}{1.00} & \multicolumn{2}{|c|}{1.10} & \multicolumn{2}{|c|}{1.15} \\
\hline & Core Set & Accuracy (\%) & Core Set & Accuracy (\%) & Core Set & Accuracy (\%) \\
\hline $\mathrm{C} 4.5$ & $\begin{array}{l}\text { Level of injury, } \\
\text { smoking, exercise }\end{array}$ & 56.82 & $\begin{array}{l}\text { Level of injury, } \\
\text { smoking, duration } \\
\text { of injury }\end{array}$ & 50.76 & $\begin{array}{l}\text { Level of injury, } \\
\text { smoking, duration } \\
\text { of injury, BMI, } \\
\text { age }\end{array}$ & 69.70 \\
\hline
\end{tabular}


(a)

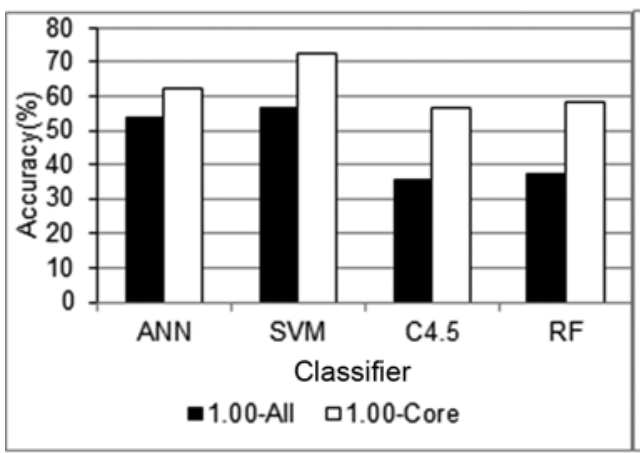

(b)

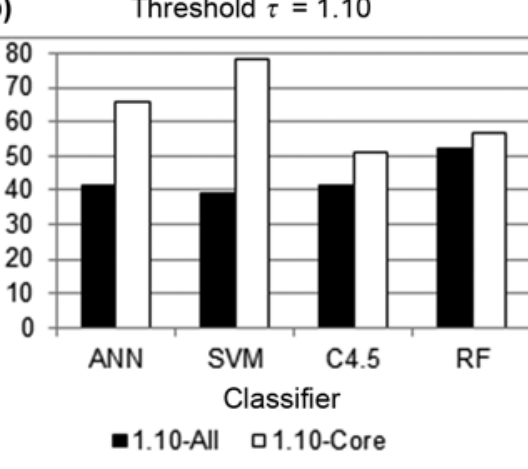

(c)

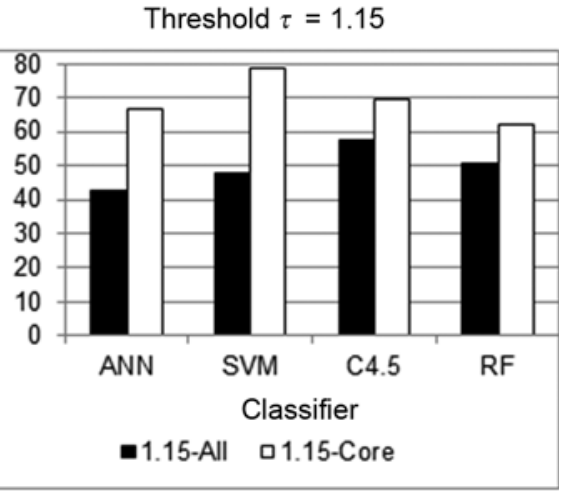

Figure 4.

Accuracy comparisons between use of all attributes and of core attributes for thresholds (a) 1.00, (b) 1.10, and (c) 1.15. ANN = artificial neural network, RF = random forest, SVM = support vector machine.

attributes to further improve the intelligent model's prediction accuracy.

Specifically, we modeled research participants with their demographic information, neurological functions, and SCI injury history, which were important to depict individuals with SCI while the values could be easily obtained. We attempted to build the intelligent model with these "handy" attributes. The advantages are to enable people with SCI to easily use the intelligent model and to leave room for this model to be upgraded in future studies. Experimental results showed that the intelligent model built upon these handy attributes held the promise and achieved satisfying accuracy.

To ensure the quality of training data, i.e., to reduce the chances of false positives, we introduced two more thresholds for the skin perfusion increase ratio $\beta$ (see Equation (2)), namely, 1.10 and 1.15. The greater the threshold is, the more confidence we have when labeling the training data. The reason is that the noise or measurement precision may affect $\beta$ such that its value may be only marginally greater than 1 . Such marginal cases are more likely labeled as negative if we use thresholds of 1.10 or 1.15 . In other words, we only labeled positive for true positive cases. Table 3 and Figure 5 show that all the classifiers achieved their highest classification accuracy when using the threshold of 1.15. Such results further affirmed that the intelligent model could produce reliable outputs if a bigger threshold was used. In addition, the use of thresholds can bring another benefit: if we continue to increase the threshold value, we can interpret the positive cases as the favorable tilt and recline settings that can result in significant skin blood flow increase.

To construct the intelligent model, we investigated four popular classifiers, namely, ANN, SVM, C4.5, and RF. As a critical part of the modeling, we applied an approach, LOO, to evaluate the performance of the intelligent models built by these classifiers. This approach was more meaningful and effective than the popular RSE and 10Fold approaches because the induced intelligent model was always tested with a new (unseen) human participant. Figure 2 illustrates the effectiveness of using the LOO approach to reveal the overfitting effect. All the

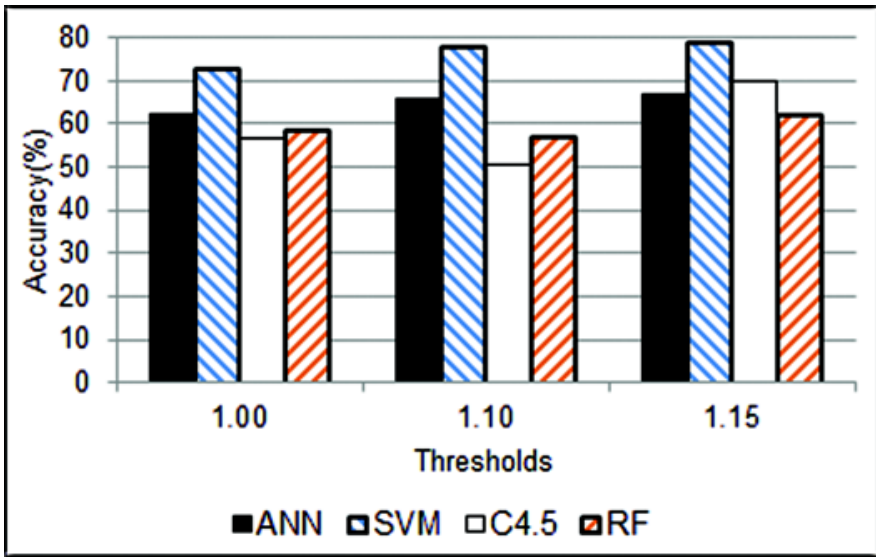

Figure 5.

Comparisons of classification accuracies in three categories when subset of attributes was used. ANN = artificial neural network, RF = random forest, SVM = support vector machine. 
classifiers sharply dropped their prediction accuracy in the experiment of LOO when all the attributes were used to model the research participants.

Then, a question naturally arises: are all the attributes useful in modeling the participants [11]? Existing research shows that (1) people with certain attributes are more vulnerable to pressure ulcers [33] and (2) irrelevant or redundant attributes may negatively affect the learning quality [32]. To answer this question in our study, we proposed a two-phase feature selection algorithm. In the first phase, a well-known feature selection algorithm, CFS [29], was used to identify the initial core attributes. Then, the second phase algorithm iteratively added to the core attribute set the attributes that could significantly improve the prediction accuracy by performing the LOO experiment. The advantages of this two-phase feature selection algorithm are manyfold. First, as the second phase attempts to improve the prediction accuracy based on the result of CFS, the performance of the final model would be at least as good as the one generated by CFS. Table 3 shows that only RF used the initial core attribute set (generated by CFS) in its final model in the category of 1.00. All other experiments expanded the core attribute set and achieved higher prediction accuracy. Second, we used the actual classifiers (i.e., ANN, SVM, $\mathrm{C} 4.5$, and RF) in the second phase to identify the important attributes. Hence, when the second phase was over, the intelligent models were also constructed. Third, the cost of acquiring training data will be lowered when using a subset of attributes; meanwhile, all the classifiers significantly improved their prediction accuracy in all three categories as shown in Table 3 and Figure 4. Fourth, this approach makes it possible to study the relationship among participant's attributes, wheelchair tilt and recline settings, and the skin blood flow response. Future research along this direction will enable clinicians to understand how individual's attributes may relate to the efficacy of tilt and recline usage and consequently provide effective guidelines to their patients.

With the subset of attributes obtained by using our two-phase feature selection algorithm, SVM outperformed all other classifiers in all three categories. It achieved the highest accuracy (78.79\%) when using the threshold of 1.15, as shown in Table 3. This result confirmed Vapnik's claim that SVM often returns global optimal results [23]. ANN also demonstrated stable classification and generalization ability. Its overall performance could be ranked second. However, when all the attributes were used, C4.5 and RF performed relatively better in LOO: RF achieved the highest accuracy in the category of 1.10 and $\mathrm{C} 4.5$ achieved the highest accuracy in the category of 1.15 (Table 2(c)). Part of the reason is that RF and C4.5 only used a subset of the attributes to classify data. Hence, they may be affected less seriously by irrelevant attributes.

Besides accuracy, we also employed the metrics of precision and recall to evaluate the classifiers' performance on LOO with a subset of attributes (Table 4). Precision can be defined as $t_{c} / p_{c}$, where $p_{c}$ is the number of examples that a classifier predicts to be in class $c$ (e.g., $c=$ 0 or 1$)$ and $t_{c}$ is the number of examples that the classifier correctly predicts in class $c$. Recall can be defined as $t_{c} /|c|$, where $|c|$ denotes the total number of examples truly belonging to class $c$. Hence, the higher the precision and recall the better. Table 4 shows the evaluation results, which are consistent with the results on the accuracy evaluation in Table 3. SVM had high precision and recall in all three categories. ANN had the highest precision in the category of 1.00 and second best recall in 1.00 and 1.10. It also outperformed RF in most of the experiments. Combining all the evaluations, it is clear that we should focus on SVM and ANN in the future study. Especially, research has shown that ANN can induce arbitrary functions approximating to arbitrary accuracy with up to two hidden layers [10].

Table 4.

Precision and recall based on subsets of attributes.

\begin{tabular}{|c|c|c|c|c|c|c|}
\hline \multirow{3}{*}{ Algorithm } & \multicolumn{6}{|c|}{ Threshold } \\
\hline & \multicolumn{2}{|c|}{1.00} & \multicolumn{2}{|c|}{1.10} & \multicolumn{2}{|c|}{1.15} \\
\hline & Precision & Recall & Precision & Recall & Precision & Recal \\
\hline SVM & 0.730 & 0.727 & 0.854 & 0.780 & 0.876 & 0.788 \\
\hline $\mathrm{C} 4.5$ & 0.531 & 0.568 & 0.516 & 0.507 & 0.780 & 0.697 \\
\hline
\end{tabular}

$\mathrm{ANN}=$ artificial neural network, $\mathrm{RF}=$ random forest, $\mathrm{SVM}=$ support vector machine. 
Table 5.

Example of personalized guidance for one research participant, including participant's attributes, skin perfusion ration for six testing conditions, expected classification of skin perfusion ratios, and actual predicted classification.

\begin{tabular}{|c|c|c|c|c|c|c|}
\hline \multicolumn{7}{|c|}{ Personal Information $(p)$} \\
\hline Level of Injury & Smoking & Age & & Alcohol & & BMI \\
\hline C6 & No & $\mathrm{xxx}^{*}$ & & No & & 24.46 \\
\hline \multicolumn{7}{|c|}{ Skin Perfusion Information and Predictions } \\
\hline Measure & $\left(15^{\circ}, 100^{\circ}\right)$ & $\left(25^{\circ}, 100^{\circ}\right)$ & $\left(35^{\circ}, 100^{\circ}\right)$ & $\left(15^{\circ}, 120^{\circ}\right)$ & $\left(25^{\circ}, 120^{\circ}\right)$ & $\left(35^{\circ}, 120^{\circ}\right)$ \\
\hline Skin Perfusion Increase Ratio & 1.03 & 1.22 & 1.09 & 1.43 & 1.63 & 1.31 \\
\hline Expected Classification $(\tau=1.15)$ & 0 & 1 & 0 & 1 & 1 & 1 \\
\hline $\begin{array}{l}\text { Actual Predicted Classification: } \\
f(p, t, r)=\end{array}$ & 0 & 0 & 0 & 1 & 1 & 1 \\
\hline
\end{tabular}

\section{Personalized Guidance on Wheelchair Tilt and Recline Usage}

The personalized guidance is achieved by using the function $f$ (Equation (1)), which can determine whether a given tilt and recline setting is favorable for increasing a person's skin perfusion. If we apply the function $f$ to clinically recommended tilt and recline settings, we will be able to know which settings are favorable for a particular person. Table 5 shows an example of personalized guidance. The top portion of the table displays the person's information $(p)$. The bottom portion of the table illustrates the skin perfusion ratios, the expected classification for the skin perfusion ratios, and the predictions made by function $f$. According to function $f$, the set of favorable tilt and recline settings for person $p$ is $\left\{\left(15^{\circ}, 120^{\circ}\right),\left(25^{\circ}, 120^{\circ}\right)\right.$, $\left.\left(35^{\circ}, 120^{\circ}\right)\right\}$. Although function $f$ misclassified $\left(25^{\circ}\right.$, $\left.100^{\circ}\right)$, it correctly classified all other positive cases, which are more favorable than the misclassified one because they have larger skin perfusion ratios.

Formally, the personalized guidance is achieved through obtaining a set of favorable tilt and recline settings for a person $p$, which is shown as follows (Equation 8):

$$
\{\langle t, r\rangle \mid f(p, t, r)=1 \text { and }\langle t, r\rangle \in \Gamma\},
$$

where $\Gamma$ is the set of clinically recommended tilt and recline settings.

\section{Study Limitations}

First, we considered 13 attributes in this study, including demographic, neurological, and spinal injury history attributes. Other attributes, such as medication and biological attributes, may affect the results as well.
We will consider more attributes that may affect the learning quality in the subsequent study. Second, the function $f$ constructed by the classification algorithms can classify whether a tilt and recline setting is favorable for skin perfusion increase. It cannot predict the extent of skin perfusion increase, i.e., the value of $\beta$ defined in Equation 2. In the subsequent study, we will develop a learning algorithm to obtain another function $g$ that can predict the value of $\beta$. Then, we will be able to use functions $f$ and $g$ to crossvalidate the outputs of each other, i.e., a tilt and recline setting $\langle t, r\rangle$ is predicted to be positive for a patient with SCI $\left\langle a_{1}, a_{2}, \ldots, a_{k}\right\rangle$ if and only if $f\left(a_{1}, a_{2}, \ldots, a_{k}, t, r\right)=1$ and $g\left(a_{1}, a_{2} \ldots a_{k}, t, r\right)>\tau$, where $\tau$ is the threshold (e.g., 1.10, 1.15, etc.). Therefore, the prediction quality will be further improved.

\section{CONCLUSIONS}

Our study demonstrated that it is feasible to use machine learning techniques to construct an intelligent model to provide personalized guidance on wheelchair tilt and recline usage to individuals with SCI. The intelligent model achieved satisfactory accuracy by considering handy attributes of the research participants, which do not require using advanced clinical devices to obtain the values. Our two-phase feature selection algorithm could effectively refine the participant model through identifying a subset of important attributes. As a result, the intelligent model's predication accuracy was further improved. Among the classifiers we used, SVM and ANN demonstrated stable performance and will be used in the future study. 
Our long-term goal is to construct an intelligent system that models SCI individuals with their demographic, neurological, biological, medical attributes, etc. In addition to classifying favorable wheelchair tilt and recline settings, the intelligent system will also predict (1) the best tilt and recline settings that can result in the most significant skin perfusion increase and (2) the optimal duration and frequency to perform wheelchair tilt and recline to effectively reduce pressure ulcer risks.

\section{ACKNOWLEDGMENTS}

\author{
Author Contributions: \\ Data analysis and interpretation: J. Fu, M. Jones, Y. Jan. \\ Design and development of the intelligent model: J. Fu, Y. Jan. \\ Funding acquisition: J. Fu, M. Jones. \\ Manuscript preparation: J. Fu, M. Jones, Y. Jan. \\ Subject recruitment and data collection: Y. Jan \\ Financial Disclosures: The authors have declared that no competing \\ interests exist. \\ Funding/Support: This material is based on work supported by the \\ National Institute of General Medical Sciences of the National Insti- \\ tutes of Health (grant 8P20GM103447).
}

\section{REFERENCES}

1. Byrne DW, Salzberg CA. Major risk factors for pressure ulcers in the spinal cord disabled: A literature review. Spinal Cord. 1996;34(5):255-63. [PMID:8963971]

http://dx.doi.org/10.1038/sc.1996.46

2. National Spinal Cord Injury Statistical Center. Annual report for the Spinal Cord Injury Model Systems (public version). Birmingham (AL): University of Alabama; 2006.

3. Reddy M, Gill SS, Rochon PA. Preventing pressure ulcers: A systematic review. JAMA. 2006;296(8):974-84. [PMID:16926357] http://dx.doi.org/10.1001/jama.296.8.974

4. Dicianno BE, Arva J, Lieberman JM, Schmeler MR, Souza A, Phillips K, Lange M, Cooper R, Davis K, Betz KL. RESNA position on the application of tilt, recline, and elevating legrests for wheelchairs. Assist Technol. 2009;21(1): 13-22, quiz 24. [PMID:19719059]

http://dx.doi.org/10.1080/10400430902945769

5. Aissaoui R, Kauffmann C, Dansereau J, de Guise JA. Analysis of pressure distribution at the body-seat interface in able-bodied and paraplegic subjects using a deformable active contour algorithm. Med Eng Phys. 2001;23(6):359-67. [PMID:11551812] http://dx.doi.org/10.1016/S1350-4533(01)00052-2
6. Makhsous M, Priebe M, Bankard J, Rowles D, Zeigler M, Chen D, Lin F. Measuring tissue perfusion during pressure relief maneuvers: Insights into preventing pressure ulcers. J Spinal Cord Med. 2007;30(5):497-507. [PMID:18092567]

7. Hobson DA. Seating and mobility for the severely disabled. In: Smith RV, Leslie JH Jr, editors. Rehabilitation engineering. Boca Raton (FL): CRC Press; 1990.

8. Hand DJ. Data mining: Statistics and more? Am Stat. 1998;52:112-18.

9. Lin RH. An intelligent model for liver disease diagnosis. Artif Intell Med. 2009;47(1):53-62. [PMID:19540738] http://dx.doi.org/10.1016/j.artmed.2009.05.005

10. Mitchell TM. Machine learning. New York (NY): McGraw-Hill; 1997.

11. Larrañaga P, Calvo B, Santana R, Bielza C, Galdiano J, Inza I, Lozano JA, Armañanzas R, Santafé G, Pérez A, Robles V. Machine learning in bioinformatics. Brief Bioinform. 2006;7(1):86-112. [PMID:16761367] http://dx.doi.org/10.1093/bib/bbk007

12. Ezziane Z. Applications of artificial intelligence in bioinformatics: A review. Expert Syst Appl. 2006;30:2-10. http://dx.doi.org/10.1016/j.eswa.2005.09.042

13. Djavan B, Remzi M, Zlotta A, Seitz C, Snow P, Marberger M. Novel artificial neural network for early detection of prostate cancer. J Clin Oncol. 2002;20(4):921-29. [PMID:11844812] http://dx.doi.org/10.1200/JCO.20.4.921

14. Michiels S, Koscielny S, Hill C. Prediction of cancer outcome with microarrays: A multiple random validation strategy. Lancet. 2005;365(9458):488-92.

[PMID:15705458] http://dx.doi.org/10.1016/S0140-6736(05)17866-0

15. Chou SM, Lee TS, Shao YE, Chen IF. Mining the breast cancer pattern using artificial neural networks and multivariate adaptive regression splines. Expert Syst Appl. 2004;27:133-42.

http://dx.doi.org/10.1016/j.eswa.2003.12.013

16. Jovic A, Bogunovic N. Electrocardiogram analysis using a combination of statistical, geometric, and nonlinear heart rate variability features. Artif Intell Med. 2011;51(3):175-86. [PMID:20980134] http://dx.doi.org/10.1016/j.artmed.2010.09.005

17. Jan YK, Jones MA, Rabadi MH, Foreman RD, Thiessen A. Effect of wheelchair tilt-in-space and recline angles on skin perfusion over the ischial tuberosity in people with spinal cord injury. Arch Phys Med Rehabil. 2010;91(11):1758-64. [PMID:21044723] http://dx.doi.org/10.1016/j.apmr.2010.07.227

18. Jan YK, Liao F, Jones MA, Rice LA, Tisdell T. Effect of durations of wheelchair tilt-in-space and recline on skin perfusion over the ischial tuberosity in people with spinal 
cord injury. Arch Phys Med Rehabil. 2013;94(4):667-72. [PMID:23178540] http://dx.doi.org/10.1016/j.apmr.2012.11.019

19. Sonenblum SE, Sprigle SH. The impact of tilting on blood flow and localized tissue loading. J Tissue Viability. 2011; 20(1):3-13. [PMID:21145240] http://dx.doi.org/10.1016/j.jtv.2010.10.001

20. Nixon J, Cranny G, Bond S. Pathology, diagnosis, and classification of pressure ulcers: comparing clinical and imaging techniques. Wound Repair Regen. 2005;13(4):365-72. [PMID:16008725] http://dx.doi.org/10.1111/j.1067-1927.2005.130403.x

21. Jan YK, David MB. Technology for pressure ulcer prevention. Top Spinal Cord Inj Rehabil. 2006;11:30-41. http://dx.doi.org/10.1310/26R8-UNHJ-DXJ5-XG7W

22. Liao F, Burns S, Jan YK. Skin blood flow dynamics and its role in pressure ulcers. J Tissue Viability. 2013;22(2):25-36. [PMID:23602509] http://dx.doi.org/10.1016/j.jtv.2013.03.001

23. Vapnik VN. The nature of statistical learning theory. 2nd ed. New York (NY): Springer; 2000.

24. Quinlan JR. C4.5: Programs for machine learning. San Mateo (CA): Morgan Kaufmann Publishers; 1993.

25. Breiman L. Random forests. Mach Learn. 2001;45:5-32. http://dx.doi.org/10.1023/A:1010933404324

26. Alba E, Chicano JF. Training neural networks with GA hybrid algorithms. Proceedings of the Genetics and Evolutionary Computation Conference; 2004 Jun 26-30; Seattle, WA.

27. Burges CJ. A tutorial on support vector machines for pattern recognition. Data Min Knowl Discov. 1998;2:121-67. http://dx.doi.org/10.1023/A:1009715923555

28. McLachlan GJ, Do KA, Ambroise C. Analyzing microarray gene expression data. Hoboken (NJ): Wiley-Interscience, 2004.

29. Hall MA. Correlation-based feature selection for discrete and numeric class machine learning. Proceedings of the 17th International Conference on Machine Learning; 2000; Stanford, CA.

30. Witten IH, Frank E. Data mining: Practical machine learning tools and techniques. 2nd ed. San Francisco (CA): Morgan Kaufmann, 2005.

31. Rumelhart DE, Hinton GE, Williams RJ. Learning representations by back-propagating errors. Nature. 1986;323: 533-36. http://dx.doi.org/10.1038/323533a0

32. Guyon I, Elisseeff A. An introduction to variable and feature selection. J Mach Learn Res. 2003;3:1157-82.

33. Garber SL, Rintala DH, Hart KA, Fuhrer MJ. Pressure ulcer risk in spinal cord injury: Predictors of ulcer status over 3 years. Arch Phys Med Rehabil. 2000;81(4):465-71. [PMID:10768537] http://dx.doi.org/10.1053/mr.2000.3889

Submitted for publication September 11, 2013. Accepted in revised form December 30, 2013.

This article and any supplementary material should be cited as follows:

Fu J, Jones M, Jan Y. Development of intelligent model for personalized guidance on wheelchair tilt and recline usage for people with spinal cord injury: Methodology and preliminary report. J Rehabil Res Dev. 2014;51(5): 775-88.

http://dx.doi.org/10.1682/JRRD.2013.09.0199

ResearcherID/ORCID: Jicheng Fu, PhD: J-3110-2013; Maria Jones, PT, PhD: G-7519-2014; Yih-Kuen Jan, PT, PhD: A-5374-2013

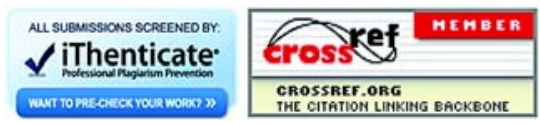


